\title{
HIJOS DE ESCLAVAS EN CÓRDOBA (ARGENTINA). UNA APROXIMACIÓN AL CICLO REPRODUCTIVO A PARTIR DE ACTAS DE BAUTISMOS
}

\author{
Sonia E. Colantonio*, María del C. Ferreyra y Dora E. Celton \\ Centro de Investigaciones y Estudios en Cultura y Sociedad (CIECS, CONICET-UNC). Córdoba. Argentina
}

PALABRAS CLAVE esclavas; características reproductivas; Córdoba; Argentina

\begin{abstract}
RESUMEN En épocas coloniales y a principios del período independentista la ciudad de Córdoba albergó una considerable población esclava, fundamentalmente mujeres provenientes de diferentes grupos etno-sociales que servían la mayoría de las veces en hogares de blancos. Contando con una amplia base de bautismos de hijos de esclavas realizados en la iglesia Catedral, nuestro objetivo fue estimar y analizar a la luz de su condición jurídica distintos indicadores de su ciclo reproductivo. Comparando las mujeres casadas con las solteras, dada la elevada ilegitimidad en los nacimientos, se estimaron la edad media al primer matrimonio, al primer
\end{abstract}

hijo y al último, el número medio de hijos nacidos vivos, el intervalo protogenésico y el intergenésico según la paridad. Los resultados muestran que las esclavas se reproducían tanto casadas como solteras aunque comenzando predominantemente fuera del matrimonio, en edades similares a la población blanca, el número medio de hijos nacidos vivos era de alrededor de 5, con intervalos proto e intergenésicos largos. Estas características habrían respondido fuertemente a las condiciones particulares en que vivían y a la importancia económica que tenía durante el período la posesión de esclavos. Rev Arg Antrop Biol 17(1):35-45, 2015.

\section{KEY WORDS slave women; reproductive characteristics; Córdoba; Argentina}

ABSTRACT During the colonial and early post-Colonial period, Córdoba city was home to a considerable slave population mainly women from different ethno-social groups serving in white people's households. From a database consisting of registers of slave baptisms at the Cathedral church, indicators of slave women's reproductive cycles were analyzed considering their marital status. Given the high number of extramarital births, single and married women were compared. The mean age at first marriage, at first birth and at the last one, the mean number of live children, and the protogenesic and intergenesic intervals by parity were calculated. The results show that slave women bore children both when married and single, but starting mainly when single, at similar ages to those of the white population. The average of live births was about 5 , and proto and intergenesic intervals were long. These characteristics might have been strongly influenced by their particular life conditions and by the economic importance of having slaves during the period. Rev Arg Antrop Biol 17(1):35-45, 2015.
La esclavitud ha sido un fenómeno generalizado en la historia colonial de Argentina, extendido tanto a lo largo de las áreas rurales cuanto de las urbanas, con mayor número de esclavas mujeres en estas últimas. Tal es el caso de la ciudad de Córdoba, en la cual éstas representaron aproximadamente el $60 \%$ de la población esclava entre fines del siglo XVIII y el primer tercio del XIX. Los varones esclavos predominaban en general en el campo en razón de la demanda para actividades agrícolas. Durante el período revolucionario y de consolidación de la república, los esclavos pudieron cambiar de estatus jurídico siguiendo nuevas modalidades de liberación con las nuevas leyes (Crespi, 2010) comenzando con posterioridad a 1810 a formar una parte importante de los batallones que surgían de las levas y permaneciendo por muchos años en los ejércitos patrios a cambio de su libertad. Cabe destacar que en Córdoba sólo una parte de la población esclava era negra y que una importante porción de esclavos era producto de mezclas étnicas donde la madre había legado la condición de esclavitud (Celton, 1993), diciéndose que "el parto sigue al vientre", al menos hasta 1813 cuando se promulga la libertad de vientres.

A pesar de que se ha postulado para las esclavas de otras zonas del país una baja fecundidad fundamentalmente en condiciones de ilegitimidad, el caso de Córdoba no parece seguir

Financiamiento: CONICET (PIP 112-201101-00796); Secretaría de Ciencia y Técnica (SECyT), Universidad Nacional de Córdoba (UNC) (PID 05/I683).

*Correspondencia a: Correspondencia a: Sonia Colantonio. Facultad de Ciencias Exactas, Físicas y Naturales (Vicedecanato). Avenida Vélez Sarsfield 299. X5000JJC Córdoba. Argentina. E-mail: scolanto@efn.uncor.edu

Recibido 05 Mayo 2014; aceptado 08 Julio 2014

doi:10.17139/raab.2015.0017.01.06 
ese patrón. El objetivo del presente trabajo es aportar algunos indicadores del proceso reproductivo en las esclavas de Córdoba, atendiendo especialmente a la extensión del período de reproducción, la edad de las madres, paridad y los intervalos protogenésicos e intergenésicos, todo ello en relación a la condición de legitimidad e ilegitimidad.

\section{MATERIAL Y MÉTODOS}

La fuente primaria de datos son las Actas de Bautismo de Naturales de la Iglesia Catedral de la ciudad de Córdoba que comienzan en 1746, correspondientes a nacidos entre los años 1733 y 1816. El primer bautismo de hijo de esclavo fue el de una "negra Angola" llamada Úrsula, bautizada en 1757 y nacida en 1733. De las mencionadas actas se seleccionaron los bautismos de niños $(\mathrm{N}=2364)$ provenientes de 513 madres esclavas. Como fuentes secundarias, cuando no había partida de bautismo, se usaron otras tales como cartas de ventas, cartas de libertad, partidas de defunción, juicios sucesorios, algunos expedientes y partidas de matrimonio que indican la edad al casamiento del causante o las declaraciones de testigos en juicios civiles y criminales. De los datos consignados en cada acta y/o recuperados de las fuentes secundarias se utilizaron: 1) nombre (y apellido si tenía) de la madre esclava, 2) año de nacimiento, 3) año de matrimonio, 4) edad al matrimonio, 5) año de muerte de la esclava, 6) sexo del bautizado, 7) año de nacimiento del bautizado (y mes en el caso de que estuviese consignado), 8) filiación del nacido [legítimo o "natural" (ilegítimo)] y 9) edad de la madre al nacimiento del niño.

El año de nacimiento del niño es exacto en el $76,5 \%$ de los casos, "aproximado" en el 19,7\% y ausente en el 3,8\% (90 casos). El mes de nacimiento sólo figuraba en 360 casos $(15,2 \%)$, razón por la cual tanto los intervalos protogenésicos como intergenésicos sólo pudieron ser calculados con cifras enteras (años) en los casos restantes. Para el año de nacimiento de la madre, se contó con la fecha exacta en el $18 \%$ de ellas, mientras que en el $82 \%$ restante tuvo que ser estimado en forma aproximada a partir de las restantes fuentes. Respecto a la filiación de hijo "legítimo" o "natural", sólo en un 1\% (24 casos) el dato no estaba disponible, por lo que éstos se excluyeron del análisis cuando se consideró la filiación.
Con el fin de verificar posibles cambios temporales en el registro, las cohortes de bautismos se subdividieron en 4 períodos de 20 años cada uno (desde 1733 a 1816). El bautismo en la ciudad coincide aproximadamente con la fecha de nacimiento, con un intervalo promedio en españoles de 2,7 días (N:2374 casos) y en naturales 2,5 días ( $\mathrm{N}: 3798$ casos) antes del bautismo, con desviaciones estándar casi idénticas a los promedios (Ferreyra, 1998). El período de 20 años constituye también una aproximación a la duración promedio de la generación en el lapso estudiado ya que como se verá, coincide con la edad media de las madres al primer hijo (20,2 años). Cabe destacar que en los cálculos de la edad media al primer matrimonio, al primer hijo, al último, etc., se tuvo en cuenta la corrección de 0,5 según Henry (1983) para edades cumplidas.

El intervalo protogenésico se calculó para esclavas casadas considerando aquellas cuyo primer hijo nació a más de 8 meses (previendo algún nacimiento pre-término) posteriores al casamiento en los casos en que se tenía fecha con mes y año. En el resto, se consideraron las que tenían al menos diferencia de 1 año entre ambos sucesos (en numerosos casos coincidían ambas edades, seguramente porque el embarazo habría conducido a un rápido matrimonio). En lo referido a intervalos intergenésicos se siguió el mismo procedimiento. Aunque algunos estudios toman sólo los primeros 5 intervalos (Rabbi et al., 2013) en nuestra población se prefirió tomar a todos los intervalos, ya que muchas mujeres tenían un elevado número de hijos.

Aunque este estudio es sobre una población completa (todos los bautizados) en un período dado y no sería estrictamente necesario establecer si las diferencias encontradas son o no significativas, cada análisis referido a edades o tamaño de prole fue complementado con un test de $t$ y ANOVA para la comparación entre madres casadas y solteras, tanto para las diferentes edades de la madre analizadas como para el tamaño de la prole. Ello en razón de que dadas las pequeñas diferencias nuestras interpretaciones serán más confiables.

Finalmente, cabe destacar que no nos referiremos a toda la población esclava sino sólo a aquellas esclavas que bautizaron sus hijos nacidos entre 1733 y 1816 . Así, los resultados no incluirán la posible mortalidad anterior ni 
posterior al bautismo de los niños. En la base de datos están todos los primeros hijos tenidos por cada mujer, pero como no todas han completado su reproducción en el período que llega hasta 1816 , las estimaciones inherentes a ciclos reproductivos completos se realizaron con aquellas esclavas que bautizaron su primer hijo en el período y en las cuales la edad de salida de observación (es decir, la edad que tenía la mujer la última vez que se la encontró en las fuentes) fue mayor o igual a 45 años. No se tomó como fecha término de la observación la muerte del cónyuge en razón de que la reproducción en este caso no es estrictamente matrimonial debido al importante porcentaje en la población de niños nacidos fuera del matrimonio, muchos nacidos después de la muerte del esposo. Tampoco se consideró como edad límite los 49 años sino los 45, siguiendo las recomendaciones de Henry (1983) a fin de perder el menor número de proles completas.

\section{RESULTADOS Y DISCUSIÓN}

La distribución de los bautismos según el período de nacimiento muestra un aumento hasta fines del siglo XVIII y a partir de allí un descenso hasta el último año considerado (1816). Obviamente la razón principal de los bajos porcentajes en el primero y último período es que muchos nacidos antes del primero no figuran en el libro de bautismos y además algunos nacidos en el último período aún quedarían sin bautizar. No obstante sorprende el alto porcentaje en 1773-1792 (Tabla 1).

Arcondo (1993) cita que la tendencia en la evolución de los nacimientos está asociada a la de los bautismos y comprueba en éstos un crecimiento de la población, rápido y sostenido durante la segunda mitad del siglo XVIII, el que

TABLA 1. Proporción de bautismos según el periodo de nacimiento del niño

\begin{tabular}{cc}
\hline Período de nacimiento & Bautismos $(\%)$ \\
\hline $1733-1752$ & 1,1 \\
$1753-1772$ & 26,2 \\
$1773-1792$ & 47,0 \\
$1793-1816$ & 25,7 \\
\hline
\end{tabular}

atribuye a los fenómenos concomitantes de la caída de la mortalidad y el aumento de los nacimientos. La misma tendencia encuentra Celton (1994) tanto para la población blanca como la de castas, pero destaca la merma a la mitad en la población esclava especialmente a partir de 1813 debido a factores como la libertad de vientres decretada por la Asamblea de ese año, la redención de esclavos destinados al ejército y la consecuente mortalidad. Asimismo, en lo referido a ventas de esclavos en Córdoba (Celton, 2000) se registra una merma en 1795-1799, coincidente con la baja en los nacimientos registrada en este trabajo.

Una característica a destacar y no esperada en los resultados es que la filiación de los niños al nacer mostró predominancia de la legitimidad $(56,3 \%)$ respecto a la ilegitimidad $(43,7 \%)$ cuando se consideran todos los nacimientos. Esto muestra que si no generalizada, la institución matrimonial era frecuente. Klein y Vinson (2013) afirman que la Iglesia en las colonias españolas promovió el matrimonio también entre los esclavos y el derecho a preservar la unión, aún en contra de la opinión de los amos. Los trabajos realizados para Córdoba, Tucumán, Catamarca, Salta y La Rioja muestran que el matrimonio legítimo era una opción entre los esclavos, pero sin que fuese el punto de partida para la formación de la familia y la reproducción (Guzmán, 2009). La Figura 1 muestra cómo evolucionó en Córdoba esta característica a través del tiempo. Si bien en un primer momento predominan los niños ilegítimos, a medida que transcurre el tiempo los hijos legítimos de esclavas se hacen cada vez más frecuentes. Celton (2008) encuentra que en la ciudad de Córdoba durante todo este período aumentó la proporción de personas que se casaba, incluyendo aquellas de origen ilegítimo, debido a los esfuerzos del Estado y la Iglesia para promover el matrimonio sacramentalizado.

Sin embargo, si se tienen en cuenta a los primeros hijos, aparece un $67,3 \%$ de ilegítimos y un $32,7 \%$ de legítimos, lo que constituye un primer indicador de que las esclavas tendrían mayoritariamente el primer hijo en estado de soltería. De acuerdo a lo anterior, cabe la posibilidad de que a estas tendencias se sume el efecto del rango del niño; es decir que a medida que aumenta el número de partos de la madre, 


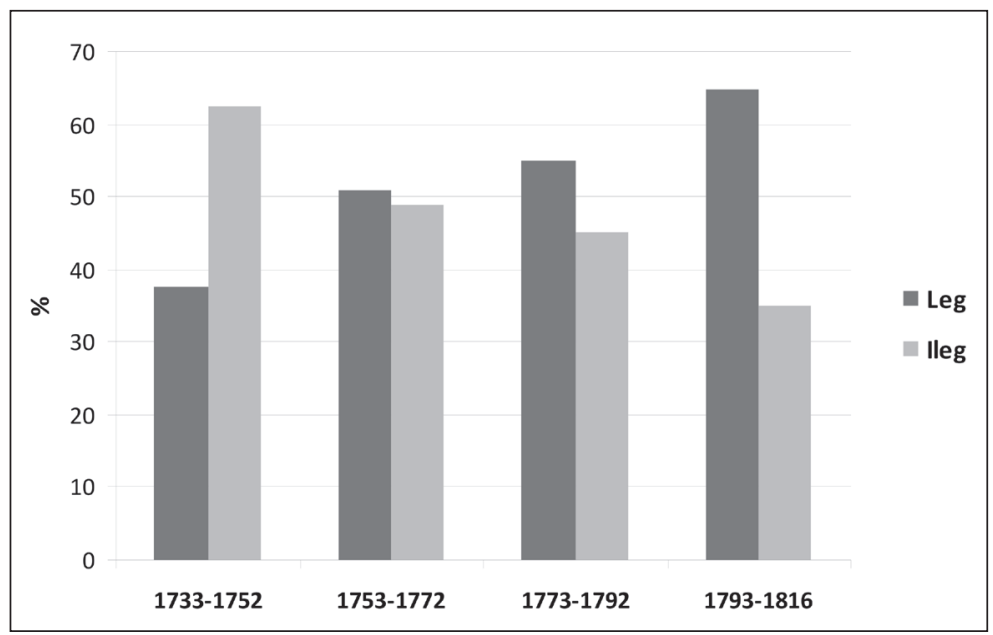

Fig. 1. Porcentajes de cada filiación (Legítimos e Ilegítimos) por período de nacimiento de los niños.

habría mayor probabilidad de que esté casada. Efectivamente, considerando todo el período con datos en la Figura 2 se observa claramente que la relación antedicha está presente y que especialmente el primer hijo pero también con frecuencia el segundo, nacen mayoritariamente en condiciones de ilegitimidad, confirmando lo obtenido previamente. Además, el coeficiente de contingencia calculado entre paridad y filiación resultó altamente significativo $(\mathrm{p}=0,000)$.

Si el análisis se invierte, es decir se analiza el peso de cada paridad dentro de cada filiación, se verifica en la Tabla 2 que en los ilegítimos predominan los de paridad 1 y 2 , el tercer hijo está representado casi por igual en ambas categorías, mientras que los de paridad 4 y más son preferentemente legítimos. Parece revelarse que si bien es frecuente ser madre soltera y especialmente tener el primer hijo en ese estado, podría haber una tendencia posterior a casarse. Esto no es extraño, ya que muchas veces los amos promovían el casamiento dentro de los límites de su propiedad a fin de retener la mano de obra y la reproducción de la misma (Ghirardi, 2004).

Los resultados indican que estas esclavas comenzaban su período reproductivo siendo tanto casadas como solteras. En las que lo iniciaban casadas, la edad media al matrimonio fue de 18,99 años, idéntica a la calculada por Tadman (1996) para esclavas del sur de Estados Unidos antes de la Guerra Civil. La media al primer hijo en nuestra población era de 21,42 años, mientras que para las que lo tuvieron solteras de 20,41 años, siendo significativa la diferencia entre ambas $(p=0,04)$. La pequeña diferencia en la edad al primer hijo, con un año de anticipación en las solteras, coincide con los valores de Celton (1993) para Córdoba, quien encuentra en el grupo 20-24 años los máximos de fecundidad. Coincidentemente, los precios máximos de venta eran en las mujeres de ese grupo de edad (Celton, 2000), lo que pone en evidencia la importancia económica de la reproducción de esta población.

La misma autora consigna para las mujeres blancas una edad al matrimonio de 21,7 (Celton,

TABLA 2. Distribución porcentual de la filiación (legítimo/ilegítimo) según la paridad

\begin{tabular}{ccc}
\hline Paridad & Legítimos (\%) & Ilegítimos (\%) \\
\hline 1 & 12,70 & 34,28 \\
2 & 17,31 & 24,20 \\
3 & 17,16 & 16,93 \\
4 & 15,57 & 11,59 \\
5 & 12,55 & 6,78 \\
6 & 10,13 & 3,44 \\
7 & 6,35 & 1,57 \\
8 & 4,16 & 0,69 \\
9 & 2,34 & 0,29 \\
10 & 0,98 & 0,22 \\
11 & 0,53 & - \\
12 & 0,23 & - \\
\hline
\end{tabular}




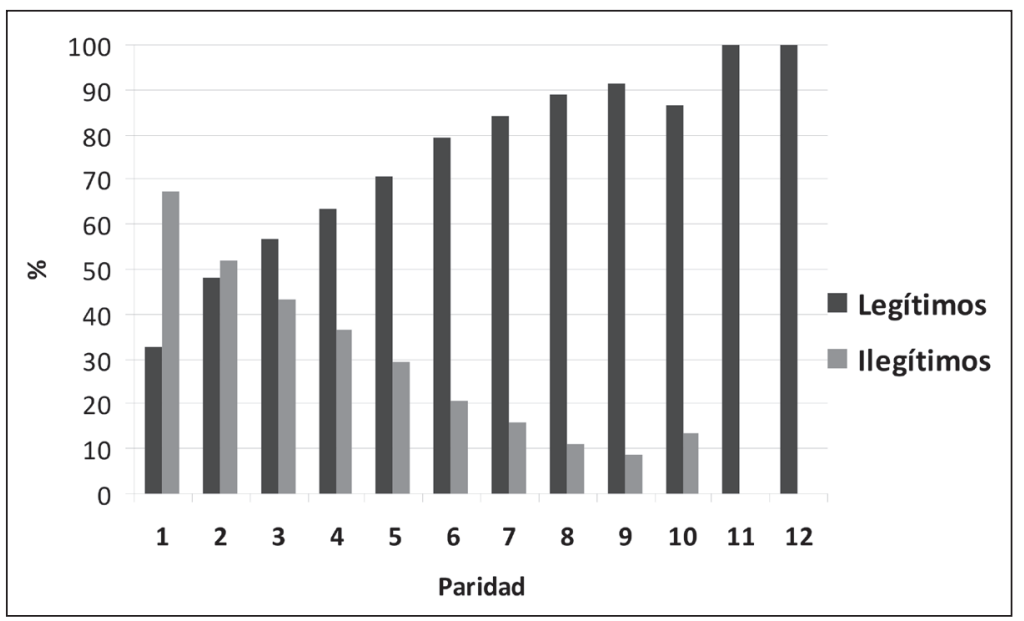

Fig. 2. Porcentajes de nacimientos por paridad (abscisas) y filiación (Leg.: Legítimos; Ileg: Ilegítimos).

2000) y aún más alta es la calculada por Ferreyra (2009) con una media de 22,2 años. En la Costa de San Isidro en el siglo XVIII (Olivero, 2006) las mujeres se casaban a los 21,4 años, aunque la autora sostiene que la edad de procreación de las de color era más baja.

Es decir, las esclavas se casaban (cuando lo hacían) y reproducían casi a las mismas edades que la población europea. Un sector de la población con reproducción temprana eran los indígenas. Klein (1986) encuentra una edad al matrimonio mucho más baja (16,8 años) en indígenas en Amatenango, México, para una época contemporánea (17851816) a la de nuestro estudio, probablemente porque la Iglesia promovía su matrimonio temprano. Desafortunadamente no disponemos de otros da- tos para las esclavas de este estudio, especialmente sobre su maduración biológica para analizar si ésta fue un condicionante en la reproducción. Un dato interesante es aportado por Trussell y Steckel (1978) para esclavas negras estadounidenses del siglo XIX, informan que tenían la menarca aproximadamente a los 15 años, luego un período infértil de 3 años y sostienen que podían concebir al cumplir 18. Si ello fuese así en las esclavas de Córdoba, es posible que la primera maternidad no estuviera condicionada por su maduración biológica, sino por factores de otra naturaleza.

En la Figura 3 se representa la distribución promedio de la edad de la madre según el rango del niño, para casadas y solteras. Como puede comprobarse en ella, ambas líneas son estre-

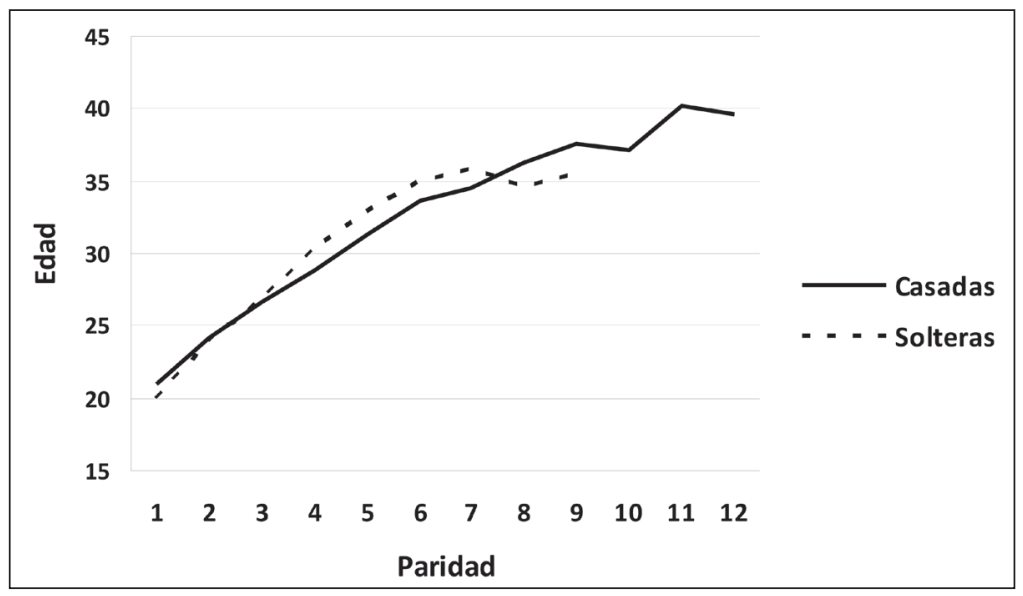

Fig. 3. Edad de la madre por paridad (abscisas) y estado civil. 
chamente cercanas, evidenciando un comportamiento similar según el estado civil.

Nos preguntamos entonces hasta cuándo proseguían estas esclavas con su reproducción. Se seleccionaron así aquellas mujeres para las cuales teníamos los bautismos desde el primer hijo y la edad de salida de observación era mayor o igual a 45 años. Si bien algunas tuvieron su último hijo a los 49 ó 50 años, la edad media al último nacimiento fue de 35,3 años para las alguna vez casadas y 33,6 años para aquellas solteras definitivas, sin diferencias significativas entre los promedios ni varianzas de ambas. Ello nos indica que la mayor diferencia entre solteras y casadas estaría en la longitud del período en que efectivamente se han reproducido, ya que las primeras tienen en promedio a sus hijos durante 12 años y las segundas en el transcurso de 14,5 años, con varianzas semejantes pero diferencia significativa en la duración media del período $(p=0,008)$. Este relativamente largo período en casadas no sorprende; en Campinas para 1872 el promedio de duración de los matrimonios era de 16 años y 8 meses y la unión finalizaba en general por muerte de uno de ellos, mostrando su fuerte estabilidad (Klein y Vinson, 2013).

Ahora bien, esos casi tres años de diferencia ¿serían los condicionantes de que las casadas tuvieran un mayor número final de descendientes? Ello no dependía sólo de los años transcurridos entre el inicio y el final de la reproducción sino también del ritmo y de los intervalos "no reproductivos".

Respecto al número medio de descendientes ("prole completa") las alguna vez casadas tuvieron una media de 5,64 hijos y las solteras 4,72 hijos, con diferencia significativa $(p=0,005)$ en sus medias de un hijo y con varianza semejante. Como se ha dicho precedentemente, las solteras empezaban a tener hijos poco antes que las casadas pero tenían en promedio menor cantidad. Ello suponiendo que no existiera mortalidad de los niños -sólo contamos con los hijos vivos al bautismo-y que todas las que comienzan a tener hijos continuaran vivas durante ese lapso. Los valores se acercan entonces estrechamente a la tasa global de fecundidad (4 hijos) calculada a partir del censo de 1778 por Celton (1993) con el método de "hijos propios", procedimiento que sí considera la influencia de la mortalidad y que por tanto ajustaría a los promedios obte- nidos en este trabajo. Resultados semejantes son provistos para una plantación de Trinidad por John (1988) en la primera década del siglo XIX, con una media de 4,6 hijos a los 49 años, con intervalos intergenésicos relativamente largos que el autor atribuye a una posible alta tasa de abortos espontáneos por la dureza del trabajo.

Si bien el número medio de hijos encontrado en esta población es más bajo que los tenidos por blancos para la prole completa sin mortalidad de 8,1 hijos a fines del siglo XVIII (Ghirardi, 2004) y tasa global de fecundidad de 5 (Celton, 1993), un no despreciable número de esclavas ha continuado teniendo hijos hasta edades avanzadas. Las mujeres que al nacimiento del hijo tenían una edad de 45 años fueron 25 , equivalente al $5 \%$ de la población. Para estas proles que se siguieron completando hasta el final del período reproductivo la media fue de 6,84 hijos (algunas inclusive con 11 ó 12 niños), la varianza de 6,64 y el valor modal de 9, llegando en las casadas a una prole media de 7,38 hijos, con desviaciones estándar de la misma magnitud que las proles que se completaron antes. Las distribuciones de la prole completa se alejan significativamente de una curva normal y responden perfectamente a una distribución de Poisson, en la cual la varianza -casi del mismo valor que la media- indica que la reproducción dentro del grupo es relativamente homogénea (Wood, 1994) y respondería a un patrón de fecundidad natural.

Desconocemos si las restantes mujeres han ejercido algún tipo control, pudiendo obedecer a un número máximo de hijos deseado (tal vez 5 según los promedios encontrados) o en el caso de las esclavas, representar un máximo de niños pasible de mantener cuando se vive en esta condición.

Aunque ha sido ampliamente reiterado que las esclavas tenían baja esperanza de vida y alta mortalidad durante el período reproductivo, las que componen la población estudiada y tienen la fecha de defunción $(\mathrm{N}=415)$, dan un promedio de 46,13 años al morir (mínimo de 18 años), por lo que podríamos pensar que una amplia mayoría alcanzó a completar su período reproductivo.

Como puede deducirse, la fecundidad de las esclavas en Córdoba no era baja. Si bien estas estimaciones se han efectuado con bautismos, el censo de 1813 muestra una gran proporción de niños esclavos, que representan el $33 \%$ de los esclavos en la ciudad (Ghirardi et al., 2010). Tal vez haya 
una conjunción de comportamientos propios de las esclavas y otros deseados por los amos. Aparentemente fue distinto en Buenos Aires, ya que Goldberg y Mallo (1994) hablan de las causas de la baja fecundidad esclava. Para otros países (Estados Unidos, Cuba, Brasil y Guayana Británica) Klein (1986) sostiene que la tasa de fertilidad en esclavas era equiparable a la de mujeres europeas. En efecto, para esclavas colombianas Chandler (1981) da un promedio de 5 hijos a los 33 años (aunque la mitad muere luego). Recuérdese que en Córdoba el precio de las mujeres superaba al de los varones, por su papel en el hogar y porque era su fecundabilidad la que aseguraba nuevos esclavos sin importar la identidad del padre (Celton, 1993). En otros contextos, tal como las plantaciones de azúcar de las colonias inglesas, el precio de los niños era más alto y la política de los amos era propiciar los nacimientos (Tadman, 1996).

Ahora bien, el número medio de hijos ha dependido también del "pace" o ritmo con el cual se tienen y los intervalos sin hijos, es decir el período entre el matrimonio (o la madurez sexual o el inicio de las relaciones sexuales) y el nacimiento del primer niño y el período entre nacimientos sucesivos. Es obvio que en la población en estudio no puede conocerse la época de inicio de las relaciones sexuales ni tomarse como límite significativo el matrimonio ya que como se vio, más de la mitad de las madres han tenido su primer hijo estando solteras. Ello difiere de poblaciones con alta nupcialidad y una fuerte relación entre el matrimonio y el primer nacimiento, como encuentran por ejemplo Hernanz et al. (2010) para épocas actuales en Granada.

Considerando las casadas donde constaba el mes de matrimonio y el mes de nacimiento del hijo y que además tuvieron el primero más allá de los 8 meses de estar casadas, el intervalo protogenésico fue de 2,97 años, es decir que esperaron aproximadamente 3 años (se excluyó un caso anómalo de 22 años de intervalo protogenésico). Este intervalo protogenésico es mucho mayor que el encontrado por Olivero (2006) en Buenos Aires para mujeres blancas (22,4 meses), mientras los intervalos intergenésicos medios posteriores son de 3 años. Estos sí son similares a nuestro valor promedio (para todos los intervalos y todas las madres) que resultó de 3,4 años, pero obviamente variando según la paridad ya que en general, la longitud del intervalo a la concepción decrece con el incremento de la paridad (Singh et al., 2011). Los valores hallados se alejan sustancialmente de lo que se considera actualmente el "óptimo" (de 18 a 23 meses). Cuando es menor a 20 meses, puede pensarse que la frecuencia de lactancia natural es baja. Sugestivamente, en los países menos desarrollados es de aproximadamente tres años y más corto en poblaciones con buen estado de nutrición. La Figura 4 muestra que el intervalo 2 (entre el primero y segundo hijo) fue el más largo (3,96 años), incluso mayor que el protogenésico y sólo igua-

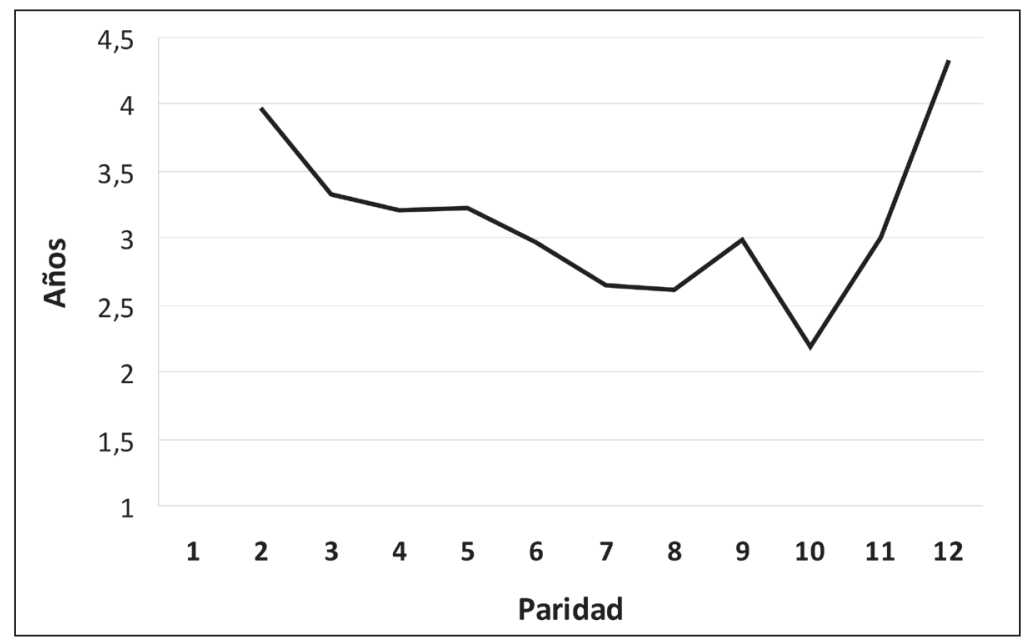

Fig. 4. Intervalo (en años) entre paridades sucesivas. Cada punto indica la diferencia con el hijo anterior. 
lado en las esclavas por unos pocos intervalos entre el hijo $11^{\circ}$ y el $12^{\circ}$.

En los que nacieron en condiciones de legitimidad el intervalo 1-2 es de 3,7 años, mientras en los hijos naturales es de 4,2 , pero sin diferencia significativa entre las medias y las varianzas. Esa pequeña diferencia podría estar asociada a factores tales como la inseguridad de estar sin pareja estable y demorar todo lo posible un segundo hijo o que luego del primero intentaran casarse, mientras postergaban el segundo. Esta hipótesis concuerda con la explicación dada por Klein y Vinson (2013) quienes aunque refiriéndose a Brasil, dicen que eran muy frecuentes las relaciones premaritales de las esclavas y que luego del primer hijo, la mujer entraba en relación estable fuese o no el padre del niño. Lo corriente era el recambio entre el primero y segundo hijo y que habitualmente el segundo (o tercero) era inscripto como legítimo. Citan además que las casadas tenían sus hijos más seguido, lo que también coincide con la población de esclavas estudiadas, donde las alguna vez casadas tenían un intervalo promedio de 3,2 años y las solteras de 3,8 años, con diferencias significativas entre sus medias $(\mathrm{p}=0,00)$.

Con respecto al intervalo intergenésico promedio, el ANOVA por rango indicó diferencias altamente significativas $(p=0,00)$ entre las distintas paridades. El test a posteriori $\mathrm{C}$ de Dunnet mostró efectivamente semejanza entre el intervalo 2 y los intervalos 9, 10, 11 y 12 por un lado y entre el 3 y el 8 por el otro (Fig. 4). Es decir, son largos los últimos, como es de esperar en mujeres que por diversas razones quieren limitar (o postergar) los hijos tenidos y en el caso de las esclavas motivo de este estudio también el intervalo 2, probablemente relacionado a la pauta mencionada de búsqueda de una relación estable. Un primer intervalo más largo es observado habitualmente en regímenes de matrimonio temprano y una asociación negativa con la edad al casarse (Amin y Bajracharya, 2011), pero el caso de la población de esclavas analizada no responde a la baja edad ni presenta dicha correlación significativa, seguramente porque como se dijo, responde a otras pautas.

Una característica también propia del ciclo reproductivo de las esclavas de Córdoba surgió al comparar el intervalo intergenésico según el sexo del hijo anterior, ya que en general la preferencia por los varones aumenta el intervalo posterior a su nacimiento y la duración del cuidado materno. Por ejemplo, Begna et al. (2013) en Etiopía encuentran que intervalos largos conducen a un embarazo a término y mayor crecimiento del niño, con una lactancia más larga. Si el hijo previo era niña (con menor valoración cultural) tendían a acortar el intervalo. En Córdoba, donde era de suma importancia el esclavo para el trabajo doméstico aparece el fenómeno opuesto. Aunque sin diferencias significativas entre sexos, el intervalo posterior al nacimiento fue la mayoría de las veces más largo cuando se trató de una niña (hasta la paridad 6).

Recapitulando lo encontrado acerca de las proles completas, todo lo anterior indica que la diferencia de un hijo más en las esclavas casadas resultaría de la sumatoria de las diferencias no significativas que aparecen en cada uno de los intervalos intergenésicos, pero que finalmente resulta en un lapso diferencial entre el primero y el último hijo.

Asimismo, en la población esclava los tiempos de espera eran largos (protogenésico 3 años, intergenésico 1-2 de 4 años y promedio de intervalos intergenésicos de 3,4 años). Cabe preguntarse cuáles podrían haber sido los factores condicionantes. No tenemos otros datos de estas esclavas como para averiguarlo, pero puede pensarse en algunas causas que las afectaran. Una de ellas es su común empleo (a veces obligación) como "amas de leche". Muchos son los relatos acerca de esclavas amamantando a un hijo ajeno y al propio o a veces restringiendo a este último el pecho por la obligación de alimentar al blanco o por ganar dinero trabajando como nodriza ( $\mathrm{Ci}$ vantos, 2005, entre otros). Es sabido que el amamantamiento constituye un importante factor que afecta la longitud de los intervalos intergenésicos, tanto en poblaciones con o sin contracepción (Montgomery y Cohen, 1998). Al respecto, Klein y Vinson (2013) encontraron muy altas tasas de natalidad en esclavas de Estados Unidos, que amamantaban a sus hijos un año, contra los dos años (la lactancia promedio en Africa occidental) que era frecuente en otras esclavas latinoamericanas, lo que probablemente aumentaba la sobrevivencia de los niños. En la población esclava del territorio argentino los niños podían permanecer en general con su madre sólo dos años (Crespi, 2010) y con posterioridad su destino era el 
dispuesto por el amo hasta la mayoría de edad, pudiendo ser vendido a los 4-5 años (Ferreyra, 2012). Si esos primeros años se usaban para amamantar pueden explicarse los extensos intervalos intergenésicos aquí encontrados; si además se suma la función de ama de leche es muy posible que se prolongaran los períodos de amenorrea y los intervalos intergenésicos. Como muestra de la importancia de la lactancia en esclavas vale como ejemplo los relatos de Bonnell Phillips (1918) sobre que a las esclavas que amamantaban en las plantaciones se les permitía estar en sus hogares hasta el amanecer antes de ir al campo y durante 20 meses. El lugar de trabajo debía quedar cerca del hogar, podían descansar y refrescarse antes de amamantar, tenían 45 minutos para estar con el niño y retornaban al hogar tres veces al día. Otra variable que afecta los intervalos intergenésicos es la mortalidad del niño. En regímenes de fecundidad natural donde el amamantamiento es regla, la muerte del niño suspende la amenorrea lactacional y se vuelve a concebir (John, 1988). La mayoría de los estudios sobre esclavitud coinciden en postular altas tasas de mortalidad neonatal e infantil que podrían haber influido en los intervalos intergenésicos pero tal vez sin acortarlos significativamente.

También como posible condicionante para los largos intervalos y a pesar de ello, lograr una prole relativamente numerosa puede mencionarse el trabajo forzado al que generalmente estaban sometidas y la capacidad de reponerse luego del nacimiento y la crianza de uno o más niños. El trabajo y la situación de las esclavas domésticas, como el caso de Córdoba, eran menos nocivos que los de trabajadoras en minas y haciendas, pero desconocemos la influencia de ese factor en la población estudiada. Otra vez pensamos tanto en variables maternas como en otras externas y de naturaleza diferente. Seguramente el tipo de trabajo y el amamantamiento pueden haber alargado los intervalos, pero el interés por parte del blanco de producir más esclavos tal vez haya influido para lograr una prole numerosa. Por ejemplo Klein (1986) sostiene que aunque el estado físico y la dieta en regímenes de "buen trato" influyesen adelantando la menarca y postergando la menopausia, la comparación de esclavas norteamericanas con las de Antillas (en peores condiciones laborales) no mostraron diferencias en el ciclo reproductivo.

No se conoce exactamente cómo los factores biológicos y genéticos afectan los intervalos. Las mujeres varían en su capacidad de concebir y llevar a término un embarazo; la salud y el estado nutricional de la madre al inicio de la gestación afecta el resultado, la habilidad para amamantar y especialmente la salud del niño en la infancia temprana (RamaRao et al., 2006).

Aunque en la población de bautizados en Córdoba no es posible calcular ningún indicador de mortalidad infantil, sabemos que la mortalidad pre-reproductiva y las condiciones de salud, morbilidad y mortalidad en la temprana infancia son factores que definirán la segunda etapa de la biología reproductiva de la población, es decir la sobrevivencia de la próxima generación. Llegamos así a considerar el último de los condicionantes que consideramos más relevantes en la extensión de los intervalos intergenésicos, cual es el estado nutricional tanto de la madre (para recuperarse y concebir más niños) como del niño (para su supervivencia hasta la llegada de un próximo hermano). Pocos estudios exhaustivos existen al respecto y la mayoría alude a una nutrición pobre y deficiente, desbalanceada y potenciadora de múltiples enfermedades y muertes de esclavos. Un conocido trabajo cuantitativo realizado por Fogel y Engerman (1979) a través de documentos censales y otros datos, sostiene que los esclavos de plantaciones de algodón de Estados Unidos tenían dietas calórica y nutricionalmente adecuadas dado que los dueños evitaban la enfermedad y la desnutrición para aumentar su rendimiento y que su nivel era equivalente al de trabajadores blancos de la época. Más adelante Steckel (1986), con datos físicos y otros indicadores ecológicos concluyó que los esclavos americanos en su temprana infancia tenían un estado nutricional asimilable al de los más bajos de las poblaciones estudiadas por los auxólogos. Sin embargo, dado que a partir de los 10 años la situación se revierte y llegan a los estándares modernos de peso, indicaría que mientras su nutrición era pobre al inicio eran bien alimentados cuando se acercaban a la edad de trabajar. Si relacionamos estos datos con las esclavas cordobesas, quienes vivían como servicio doméstico en mejores condiciones que las de las plantaciones, podríamos pensar que ellas estarían relativamente bien nutridas (o tendrían 
posibilidades de recuperar la energía gastada en la concepción, alumbramiento y amamantamiento alargando el intervalo hasta la próxima gestación) y tal vez por ello las encontramos vivas hasta altas edades, teniendo un considerable número de niños. Pero tal vez no fueran esas las condiciones de nutrición de los niños y por ello las altas tasas de mortalidad encontradas tanto para Buenos Aires por Goldberg y Mallo (1994) como para muchos países de Hispanoamérica colonial.

Aunque todos los condicionantes mencionados son pasibles de haber actuado sobre el ciclo reproductivo de las esclavas de Córdoba, no podemos hasta el momento ponderar la importancia o la intensidad con la que habría actuado cada uno y seguramente este peso habrá sido diferente según la mujer de que se tratase, ya que las esclavas cordobesas diferían según su origen geográfico, su grupo socio-étnico de procedencia, su estado civil, el lugar donde habitaban, etc. Su conducta reproductiva, como la de muchas otras mujeres, puede enmarcarse en la teoría de los costos y beneficios derivados de tener un hijo adicional a la manera de Cordero (2009). Sin embargo, otros factores externos a las propias decisiones han debido jugar un rol determinante en el ciclo de estas mujeres debido a su condición de sometimiento a la clase blanca dominante. Si bien los beneficios de una prole numerosa pueden haber existido, sin duda los costos habrán sido particularmente más elevados para estas esclavas que eran mano de obra para la producción y un vehículo apropiado para la reproducción de la fuerza laboral.

\section{CONCLUSIONES}

Las madres esclavas de la ciudad de Córdoba aquí analizadas mostraron reproducirse tanto en condiciones de ilegitimidad cuanto dentro de un matrimonio. La legitimidad de los nacidos aumentó a través del período estudiado, pero manteniendo la pauta de que los primeros y muchas veces los segundos nacimientos se producen en estado de soltería.

Las mujeres solteras comienzan su reproducción antes que las casadas y terminan también antes, pero la sumatoria de todos los intervalos intergenésicos resulta en una diferencia en la longitud del período reproductivo de 2 años a favor de las casadas y la producción de un hijo más. A pesar de tener menos hijos que las mujeres blancas, las esclavas que se casan lo hacen aproximadamente a la misma edad que aquéllas.

La distribución del tamaño de la prole es relativamente homogénea en casadas y solteras y respondería a un patrón de fecundidad natural con una edad relativamente alta al primer hijo. El número medio de hijos nacidos vivos y bautizados sin considerar la mortalidad, es de 5,64 hijos para las alguna vez casadas y 4,72 hijos para las solteras. Una característica destacable es la considerable longitud de los intervalos sin hijos; tanto del protogenésico como de los intergenésicos, especialmente el ubicado entre el primero y segundo hijo.

Aunque no se ha estimado la mortalidad, la edad media a la muerte de las madres esclavas estudiadas indicaría que la mayoría de ellas alcanza a completar su período reproductivo.

Las características del ciclo reproductivo encontradas en las esclavas de Córdoba muestran ciertas particularidades tales como una pequeña influencia del estatus civil sobre la reproducción, mientras otros factores relacionados con su condición social de esclavas habrían actuado con mayor intensidad, tales como duración e intensidad de la lactancia alargando los intervalos intergenésicos pero que a pesar de ello, hay un número medio de hijos relativamente elevado y muchas madres sobreviven hasta el final de los años reproductivos. Aunque desconocemos hasta ahora la mortalidad de estos niños, que seguramente ha sido alta, las características de estos ciclos reproductivos habrían sido favorables al interés de los amos para que hubiese una considerable producción de nuevos esclavos.

\section{AGRADECIMIENTOS}

Los autores agradecen a las entidades que brindaron el apoyo financiero para la realización del trabajo.

\section{LITERATURA CITADA}

Amin S, Bajracharya A. 2011. Marriage and first birth intervals in early and late marrying societies: an exploration of determinants. Population Council. Annual Meetings of the Population Association of America. Washington DC. Disponible en: http://paa2011.princeton.edu/papers/111970.

Arcondo A. 1993. Mortalidad general, mortalidad epidémi- 
ca y comportamiento de la población de Córdoba durante el siglo XVIII. Desarrollo Económico 33:67-85. doi: $10.2307 / 3467340$

Begna Z, Assegid S, Kassahun W, Gerbaba M. 2013. Determinants of inter birth interval among married women living in rural pastoral communities of southern Ethiopia: a case control study. BMC Pregnancy and Childbirth 13:116. Disponible en: www.biomedcentral.com/14712393/13/116. doi: 10.1186/1471-2393-13-116

Celton D. 1993. Fecundidad de las esclavas en la Córdoba colonial. Revista de la Junta Provincial de Historia de Córdoba 15:29-49.

Celton D. 1994. Estudio demográfico de la ciudad de Córdoba durante la Gobernación Intendencia. Cuadernos de Historia. Serie Población 1:23-56.

Celton D. 2000. La venta de esclavos en Córdoba, Argentina. Cuadernos de Historia. Serie Población 2:5-21.

Celton D. 2008. Abandono de niños e ilegitimidad. Córdoba, Argentina, siglos XVIII-XIX. En: Ghirardi M, coordinadora. Familias iberoamericanas ayer y hoy. Una mirada interdisciplinaria. Asociación Latinoamericana de Población. Serie Investigaciones 2. p 231-250.

Chandler L. 1981. Family bonds and the bondsman: the slave family in Colonial Colombia. Latin American Research Review 16:107-131.

Civantos C. 2005. Pechos de leche, oro y sangre: las circulaciones del objeto y el sujeto en Cecilia Valdés. Revista Iberoamericana LXXI (211):505-519.

Cordero J. 2009. El espaciamiento de los nacimientos: una estrategia para conciliar trabajo y familia en España. Revista Española de Investigaciones Sociológicas (Reis) 128:11-33.

Crespi L. 2010. En busca de un enclave esclavista. La expedición colonizadora a las islas de Fernando Poo y Annobon, en el Golfo de Guinea. (1778-1782). Revista Digital Estudios Históricos año II (4), Uruguay. Disponible en: http://www.estudioshistoricos.org/edicion_4 / liliana-crespi.pdf

Ferreyra M del C. 1998. La ilegitimidad en la ciudad y en el campo a finales del siglo XVIII en Córdoba. En: Cambios Demográficos en América Latina: la experiencia de cinco siglos. Internacional Union for the Scientific Study of Population-Universidad Nacional de Córdoba. p 403-427.

Ferreyra M del C. 2009. Matrimonios de "españoles" en la ciudad de Córdoba en el siglo XVIII. El uso de fuentes diversas para su estudio. En: Celton D, Ghirardi M, Carbonetti A, coordinadores. Poblaciones históricas. Fuentes, métodos y líneas de investigación. Río de Janeiro. Asociación Latinoamericana de Población, Serie Investigaciones 9. p 271-299.

Ferreyra M del C. 2012. Venta de esclavos párvulos en la ciudad de Córdoba, 1700-1809. Revista Junta Provincial de Historia de Córdoba 26:37-54.

Fogel RW, Engerman SL. 1979. Recent findings in the study of slave demography and family structure. Sociology and Social Research 63:566-589.

Ghirardi MM. 2004. Matrimonios y familias en Córdoba 1700-1850. Prácticas y representaciones. Córdoba: Centro de Estudios Avanzados, Universidad Nacional de Córdoba.

Ghirardi MM, Colantonio S, Celton D. 2010. De azabache y ámbar. Tras las huellas de los esclavos de Córdoba al despuntar la revolución. En: Mallo S, Telesca I, editores. Negros de la patria. Los afrodescendientes en las luchas por la independencia en el antiguo virreinato del
Río de la Plata. Buenos Aires: Editorial SB, Colección Paradigma Indicial. p 15-38.

Goldberg M, Mallo S. 1994. La población africana en Buenos Aires y su campaña. Temas de África y Asia No 2. Facultad de Filosofía y Letras. Universidad de Buenos Aires.

Guzmán F. 2009. Representaciones familiares de las mujeres negras en el Tucumán colonial. Un análisis en torno al mundo doméstico subalterno. En: Celton D, Ghirardi M, Carbonetti A, coordinadores. Poblaciones históricas. Fuentes, métodos y líneas de investigación. Río de Janeiro. Asociación Latinoamericana de Población, Serie Investigaciones 9. p 403-425.

Hernanz JA, Acevedo Cantero P, Prado Martínez C. 2010. Socio-economic factors associated with the reproductive behaviour in rural population of the Granada province, Spain. J Hum Ecol 30:35-43.

Henry L. 1983. Manual de demografía histórica. Barcelona: Editorial Crítica.

John AM. 1988. The plantation slaves of Trinidad, 17831816: a mathematical and demographic enquiry. Cambridge: Cambridge University Press.

Klein HS. 1986. Familia y fertilidad en Amatenango, Chiapas, 1785-1816. Historia Mexicana 36:273-286.

Klein HS, Vinson B III. 2013. Historia mínima de la esclavitud en América Latina y en el Caribe. México: Editorial El Colegio de Mexico. Libro electrónico. Disponible en: http://libros.colmex.mx

Montgomery M, Cohen B. 1998. From death to birth: mortality decline and reproductive change. Washington DC: National Academic Press.

Olivero S. 2006. Natalidad y bautismo en una parroquia rural de la campaña rioplatense: la población blanca del Pago de la Costa en la primera mitad del siglo XVIII. En: Gutiérrez Escudero A, Laviana Cuetos ML, coordinadores. Estudios sobre América: siglos XVI-XX. Sevilla: Asociación Española de Americanistas. p 13171337.

Phillips UB. 1918. American negro slavery: a survey of the supply, employment and control of negro labor as determined by the plantation regime. New York: D. Appleton and Company.

Rabbi AM, Karmaker SC, Mallick SA, Sharmin S. 2013. Determinants of birth spacing and effect of birth spacing on fertility in Bangladesh. Dhaka Univ J Sc. 61:105-110. doi:10.3329/dujs.v61i1.15105

RamaRao S, Townsend J, Askew I. 2006. Correlates of inter-birth intervals: implications of optimal birth spacing strategies in Mozambique. Population Council. Disponible en: http:// www. popcouncil.org/pdfs/frontiers/ FRFinalReports/MozamOBSI.pdf

Singh NS, Singh NS, Narendra RK. 2011. Differential pattern of duration of waiting time to conception of women in Manipur. Stud Home Com Sci 5:7-12.

Steckel, R. 1986. A peculiar population: the nutrition, health and mortality of American slaves from childhood to maturity. The Journal of Economic History 46:721-741. doi:10.1017/S0022050700046842

Tadman M. 1996. Speculators and slaves: masters, traders, and slaves in the Old South. Wisconsin: University of Wisconsin Press.

Trussell J, Steckel R. 1978. The age of slaves at menarche and their first birth. J Interdiscip Hist. 8:477-505. doi: $10.2307 / 202918$

Wood JW. 1994. Dynamics of human reproduction: biology, biometry, demography. New York: Aldine De Gruyter Editions. 